\title{
Isolation and characterization of microsatellite markers for Eucommia ulmoides (Eucommiaceae), an endangered tree, using next-generation sequencing
}

\author{
W.R. Zhang ${ }^{1 *}$, Y. Li ${ }^{2 *}$, J. Zhao ${ }^{1}$, C.H. Wu ${ }^{1}, S^{\prime}$ Ye ${ }^{1}$ and W.J. Yuan ${ }^{1}$ \\ ${ }^{1}$ Institute of Chinese Materia Medica, Henan University, Kaifeng, China \\ ${ }^{2}$ College of Forestry, Henan Agricultural University, Zhengzhou, China \\ *These authors contributed equally to this study. \\ Corresponding author: W.J. Yuan \\ E-mail: lyhenau@yeah.net
}

Genet. Mol. Res. 15 (2): gmr.15027789

Received October 6, 2015

Accepted December 7, 2015

Published April 25, 2016

DOI http://dx.doi.org/10.4238/gmr.15027789

\begin{abstract}
Eucommia ulmoides Oliver, a single extant species of Eucommiaceae, is an endemic dioecious tree in China. The natural resources of E. ulmoides have rapidly declined in recent years because of the over-collection of its cortex. To design a suitable protection strategy, it is necessary to develop a set of molecular markers to investigate genetic diversity and population structure of E. ulmoides. Pyrosequencing of an enriched microsatellite library by Roche $454 \mathrm{FLX}+$ platform was used to isolate simple sequence repeats (SSRs) for E. ulmoides. A total of 1568 SSRs that contained enough flanking sequences for primer pair design were identified from 45,236 raw sequence reads. One hundred SSRs were randomly selected to design primer pairs and polymerase chain reaction was performed. Among these 100 tested primer pairs, 16 were polymorphic across 18 individuals from three E. ulmoides populations. The number of alleles ranged from 3 to 8 , with an average of 5.1. The expected heterozygosity ranged from 0.110 to 0.830 , with
\end{abstract}


an average of 0.648 , and the observed heterozygosity ranged from 0.111 to 0.833 , with an average of 0.524 . The inbreeding coefficient ranged from -0.349 to 0.547 . This set of microsatellite markers could be valuable for landscape genetic structure assessment and molecular marker-assisted breeding in E. ulmoides.

Key words: Simple sequence repeat; Genetic diversity; Population structure; Eucommia ulmoides

\section{INTRODUCTION}

Eucommia ulmoides Oliver, a single extant species of Eucommiaceae, is an endemic dioecious tree in China. Its cortex has been used in traditional Chinese medicine for over 2000 years. Previous studies revealed that the pharmacological properties of its leaves are similar to those of its cortex (Hussain et al., 2003; Chen et al., 2010). It is pharmacologically used to treat lumbago and hypertension, prevent bone senescence, and enhance liver and kidney functions. The cortex is also used as a raw material in the chemical industry for the guttapercha production. For these reasons, the cortex has been over-harvested for its high medical and industrial value. E. ulmoides is currently on the red list of endangered plant species of China (Yao et al., 2012). To design a suitable protection strategy, it is necessary to develop a set of molecular markers to investigate genetic diversity and population structure of $E$. ulmoides. Although, molecular markers such as random amplified polymorphic DNA (RAPD; Wang et al., 2006) and amplified fragment length polymorphism (AFLP; Yao et al., 2012) have been used to investigate the genetic diversity of $E$. ulmoides, simple sequence repeats (SSRs) have been considered more reliable markers owing to their high reproducibility (Li et al., 2002; Wei et al., 2013). Deng et al. (2006) first reported on microsatellite markers of $E$. ulmoides using the traditional fast isolation by AFLP sequences containing repeats protocol. However, a limited number of markers do not meet the requirements of a survey of landscape genetic structure (Hall and Beissinger, 2014). Meanwhile, because of its endangered status, artificial cultivation of E. ulmoides has been started in China. This requires higher molecular marker density to help future variety breeding.

With the development of technology, next-generation sequencing technique has widely been used for SSR isolation (Fu et al., 2014; Li et al., 2015). Next-generation sequencing can be used to obtain a large number of markers in a short time. In the present study, we report a large number of SSRs for E. ulmoides, obtained using a Roche 454 FLX+ platform.

\section{MATERIAL AND METHODS}

\section{Plant material and DNA extraction}

Fresh leaves of E. ulmoides were collected from 18 individuals belonging to three natural populations (Xinyang: $31.911^{\circ} \mathrm{N}, 114.110^{\circ} \mathrm{E}$; Nanyang: $33.619^{\circ} \mathrm{N}, 111.737^{\circ} \mathrm{E}$; Luoyang: $33.773^{\circ} \mathrm{N}$, $\left.111.627^{\circ} \mathrm{E}\right)$ from Henan Province of China. Vouchers were deposited in the herbarium of the Institute of Chinese Materia Medica at Henan University (Herbarium accession No. HCMM-101118). Total genomic DNA was extracted from fresh leaves using a plant genomic DNA extraction kit DP305 (Tiangen Biotech, Beijing, China) according to the manufacturer protocol. 


\section{DNA sequencing and microsatellite locus searching}

Approximately $2 \mu \mathrm{g}$ genomic DNA was used to construct a DNA library according to the Roche 454 GS-FLX+ library preparation protocol. Hybridization of eight biotinylated oligonucleotides $\left[(\mathrm{AG})_{10},(\mathrm{AC})_{10},(\mathrm{AAC})_{8},(\mathrm{ACG})_{8},(\mathrm{AAG})_{8},(\mathrm{ACAT})_{6},(\mathrm{ATCT})_{6}\right.$, and $\left.(\mathrm{AGG})_{8}\right]$ with the DNA library was performed to enrich the repetitive motifs. The enriched products were then sequenced using Roche $454 \mathrm{FLX}+$ platform by Personalbio (Shanghai, China). One-twelfth run was performed on the sequencing platform. The program, MISA (Thiel et al., 2003), was used to search for SSR loci with the default settings and Primer3 (Rozen and Skaletsky, 2000) was used to design primer pairs keeping the default settings.

\section{Polymerase chain reaction (PCR) and genotyping}

PCRs were performed in a $20-\mu \mathrm{L}$ reactions that contained 50 ng genomic DNA, $0.5 \mu \mathrm{M}$ each primer, and $10 \mu \mathrm{L} 2 \mathrm{X}$ Taq PCR MasterMix $(0.1 \mathrm{U}$ Taq polymerase $/ \mu \mathrm{L}, 0.5 \mathrm{mM}$ dNTPs, $20 \mathrm{mM}$ Tris-HCl, $\mathrm{pH}$ 8.3) using an S1000 Thermal cycler (Bio-Rad, USA). PCR conditions were as follows: pre-incubation at $94^{\circ} \mathrm{C}$ for $5 \mathrm{~min}$; followed by 35 cycles of denaturation at $94^{\circ} \mathrm{C}$ for $50 \mathrm{~s}$; annealing at a locus-specific temperature for $45 \mathrm{~s}$; elongation at $72^{\circ} \mathrm{C}$ for $50 \mathrm{~s}$; a final extension step at $72^{\circ} \mathrm{C}$ for $8 \mathrm{~min}$. PCR products were tested for polymorphism using $8 \%$ native polyacrylamide gels and visualized by silver nitrate staining.

\section{Data analysis}

Electropherograms for the fragment analysis were analyzed using the GeneMarker software v1.95 (SoftGenetics, State College, PA, USA). The number of alleles per locus $\left(N_{\mathrm{A}}\right)$, observed heterozygosity $\left(H_{\mathrm{O}}\right)$, expected heterozygosity $\left(H_{\mathrm{E}}\right)$, and deviations from HardyWeinberg equilibrium (HWE) were calculated with Popgene v1.32 (Yeh et al., 2000). Linkage disequilibrium (LD) between the pairs of SSR loci were calculated using GENEPOP v4.2 (Rousset, 2008). Inbreeding coefficients $\left(F_{\text {IS }}\right)$ were calculated by FSTAT v2.9.3.2 (Goudet, 1995).

\section{RESULTS AND DISCUSSION}

One-twelfth run of Roche 454 pyrosequencing yielded 45,236 raw sequence reads, ranging from 355 to $986 \mathrm{bp}$ with an average length of $573 \mathrm{bp}$. A total of 1568 SSRs were identified that contained enough flanking sequences for primer pair design. These sequences were deposited in the NCBI GenBank (GenBank accession Nos. KP248123-KP249690). One hundred SSRs were randomly selected to design primer pairs and PCR amplifications were performed. Because of the amplification of multiple bands or unsuccessful amplification of the target fragments, seventy-eight primer pairs were discarded. The remaining pairs were tested for polymorphisms using 18 individuals from three E. ulmoides populations; polymorphic amplification products were obtained in 16 individuals (Table 1). For polymorphic primer pairs, $N_{\mathrm{A}}$ per locus ranged from 3 to 8 , with an average of 5.1 (Table 1). $H_{\mathrm{E}}$ and $H_{\mathrm{O}}$ ranged from 0.110 to 0.830 (with an average of 0.648 ) and from 0.111 to 0.833 (with an average of 0.524 ) at the species level, respectively (Table 1). $F_{\text {IS }}$ varied from -0.349 to 0.547 (Table 1). Genetic diversity parameters in each population are presented in Table 2. Six SSR loci 
W.R. Zhang et al.

(DZ4,DZ7, DZ8,DZ10,DZ11, and DZ14) showed significant deviation from the HWE $(\mathrm{P}<$ $0.05)$ due to heterozygote excess or deficiency. Four pairs of loci, namely, $D Z 1$ and $D Z 4, D Z 6$ and DZ8,DZ8 and DZ14, and DZ6 and DZ16, showed significant LD, indicating significant allelic associations between these loci. This set of microsatellite markers could be valuable for landscape genetic structure assessment and molecular marker-assisted breeding in E. ulmoides.

Table 1. Primer sequences and characterization for sixteen microsatellite loci isolated from Eucommia ulmoides.

\begin{tabular}{|c|c|c|c|c|c|c|c|c|c|}
\hline $\begin{array}{l}\text { Primer } \\
\end{array}$ & Primer sequence (5'-3') & Repeat motif & $\mathrm{Ta}\left({ }^{\circ} \mathrm{C}\right)$ & Allele size (bp) & $N_{\mathrm{A}}$ & $H_{\mathrm{E}}$ & $H_{\mathrm{O}}$ & $F_{\mathrm{IS}}$ & GenBank Accession No. \\
\hline$\overline{D Z 1}$ & $\begin{array}{l}\text { F: TTACCAAAACGACATGCCAA } \\
\text { R: TTGCTGTGGGAACTGTGTGT }\end{array}$ & $(\mathrm{AAC})_{11}$ & 58 & $271-283$ & 5 & 0.732 & 0.611 & 0.169 & KP248123 \\
\hline$D Z 2$ & $\begin{array}{l}\text { F: AGCAAGAACCTGTTGCCATT } \\
\text { R: CAGTCGATAAGTTCGCCCTC }\end{array}$ & $(\mathrm{AAC})_{13}$ & 58 & $316-345$ & 8 & 0.830 & 0.833 & -0.004 & KP248129 \\
\hline$\overline{D Z 3}$ & $\begin{array}{l}\text { F: GCACAAAATTTAAGTGCGTAATC } \\
\text { R: TTCGAGAGATCATTCGTGAGA }\end{array}$ & $(\mathrm{AAC})_{13}(\mathrm{AAT})_{5}$ & 56 & $316-328$ & 7 & 0.816 & 0.722 & 0.118 & KP248130 \\
\hline DZ4 & $\begin{array}{l}\text { F: TGGATTGGGGATAATTCAATG } \\
\text { R: GAGCCAGGCTGTGATCTAGG }\end{array}$ & $(\mathrm{AAT})_{5}(\mathrm{AAC})_{21}$ & 58 & $267-282$ & 7 & 0.786 & $0.444^{*}$ & 0.441 & KP248144 \\
\hline$D Z 5$ & $\begin{array}{l}\text { F: ACGAAACATCGATCACACCA } \\
\text { R: GGGAGCGTTTCTGAAGAAGAT }\end{array}$ & $(\mathrm{AC})_{10}$ & 58 & $322-332$ & 5 & 0.713 & 0.444 & 0.383 & KP248146 \\
\hline$\overline{D Z 6}$ & $\begin{array}{l}\text { F: TTGATCCCTTGCCACAATTT } \\
\text { R: GGAACCCGTTGGATTCTACA }\end{array}$ & $(\mathrm{AC})_{10}(\mathrm{AT})_{7}(\mathrm{AG})_{8}$ & 58 & $347-353$ & 4 & 0.644 & 0.444 & 0.317 & KP248149 \\
\hline$D Z 7$ & $\begin{array}{l}\text { F: GCGTTACAAGATTTCATCGCT } \\
\text { R: AGCACCCATCGAAGAGAAAA }\end{array}$ & $(\mathrm{ACA})_{12}$ & 58 & $370-379$ & 4 & 0.729 & $0.500^{*}$ & 0.320 & KP248167 \\
\hline$\overline{D Z 8}$ & $\begin{array}{l}\text { F: CGTACCGATTTCGTATCGTG } \\
\text { R: AACATGCATGGGGTTATGGT }\end{array}$ & $(\mathrm{AG})_{11}$ & 58 & $363-371$ & 5 & 0.711 & $0.333^{*}$ & 0.538 & KP248170 \\
\hline$\overline{D Z 9}$ & $\begin{array}{l}\text { F: TTTGGAAGTTGGGAACAAAAA } \\
\text { R: AATTGATGGTTTCGGTTTCG }\end{array}$ & $(\mathrm{AAACAC})_{6}$ & 58 & $135-153$ & 4 & 0.513 & 0.444 & 0.137 & KP248175 \\
\hline$D Z 10$ & $\begin{array}{l}\text { F: GCGTGATTTGATTTGGCTCT } \\
\text { R: TGATGGCTCATTTTGGCATA }\end{array}$ & $(\mathrm{AAC})_{12}$ & 58 & $207-216$ & 5 & 0.724 & $0.333^{*}$ & 0.547 & KP248179 \\
\hline DZ11 & $\begin{array}{l}\text { F: TCACTCCTAGACCACCCACC } \\
\text { R: GGGTGTCGTTGTGTGTATGC }\end{array}$ & $(\mathrm{AC})_{13}$ & 58 & $270-278$ & 5 & 0.700 & $0.556^{*}$ & 0.211 & KP248183 \\
\hline$D Z 12$ & $\begin{array}{l}\text { F: GGATATTCGTAGCCGTTGGA } \\
\text { R: AAAACACTCGAATTGTGGGC }\end{array}$ & $(\mathrm{AC})_{19}(\mathrm{AT})_{10}$ & 58 & $220-228$ & 5 & 0.479 & 0.556 & -0.164 & KP248189 \\
\hline$\overline{D Z 13}$ & $\begin{array}{l}\text { F: TGTACGCTAAATTTTCGGGC } \\
\text { R: TCTTCTCTCGCTCTTCCTCG }\end{array}$ & $(\mathrm{AG})_{18}$ & 58 & $316-328$ & 6 & 0.792 & 0.778 & 0.019 & KP248201 \\
\hline$D Z 14$ & $\begin{array}{l}\text { F: TCACACACATACACGCATGG } \\
\text { R: TTTTGGGGTCCAAATTGTGT }\end{array}$ & $(\mathrm{CT})_{13}$ & 58 & $264-272$ & 3 & 0.624 & $0.833^{*}$ & -0.349 & KP248213 \\
\hline$D Z 15$ & $\begin{array}{l}\text { F: TACGCCTCTCATGTCCTTCA } \\
\text { R: AACTGCCCCTGACATTCTTG }\end{array}$ & $(\mathrm{GA})_{15}$ & 60 & $261-273$ & 5 & 0.470 & 0.444 & 0.056 & KP248216 \\
\hline$D Z 16$ & $\begin{array}{l}\text { F: TCAGAACATCAGCTCATCCAA } \\
\text { R: AAGGCTCGATGCCAGATAGA }\end{array}$ & $(\mathrm{GA})_{15}$ & 60 & $304-310$ & 3 & 0.110 & 0.111 & -0.015 & KP248217 \\
\hline
\end{tabular}

$\mathrm{Ta}=$ PCR annealing temperature; $N_{\mathrm{A}}=$ number of alleles; $H_{\mathrm{E}}=$ expected heterozygosity; $H_{\mathrm{O}}=$ observed heterozygosity; $F_{\text {IS }}=$ inbreeding coefficient; *Significant deviation from Hardy-Weinberg equilibrium.

Table 2. Genetic diversity parameters for three populations of Eucommia ulmoides.

\begin{tabular}{|c|c|c|c|c|c|c|c|c|c|c|c|c|}
\hline \multirow[t]{2}{*}{ Primer } & \multicolumn{4}{|c|}{ Xinyang $(\mathrm{N}=6)$} & \multicolumn{4}{|c|}{ Nanyang $(\mathrm{N}=6)$} & \multicolumn{4}{|c|}{ Luoyang $(\mathrm{N}=6)$} \\
\hline & $N_{\mathrm{A}}$ & $H_{\mathrm{E}}$ & $H_{\mathrm{O}}$ & $F_{\text {IS }}$ & $N_{\mathrm{A}}$ & $H_{\mathrm{E}}$ & $H_{0}$ & $F_{\text {IS }}$ & $N_{\mathrm{A}}$ & $H_{\mathrm{E}}$ & $H_{\mathrm{O}}$ & $F_{\text {IS }}$ \\
\hline$D Z 1$ & 5 & 0.818 & 0.833 & -0.020 & 5 & 0.742 & 0.667 & 0.111 & 4 & 0.697 & 0.333 & 0.545 \\
\hline DZ2 & 6 & 0.818 & 1.000 & -0.250 & 5 & 0.742 & 0.667 & 0.111 & 4 & 0.803 & 0.833 & -0.042 \\
\hline DZ3 & 4 & 0.803 & 0.833 & -0.042 & 4 & 0.697 & 0.667 & 0.048 & 6 & 0.803 & 0.667 & 0.184 \\
\hline DZ4 & 4 & 0.727 & $0.000^{*}$ & 1.000 & 5 & $\begin{array}{l}0.849 \\
\end{array}$ & 0.667 & 0.231 & 4 & 0.712 & 0.667 & 0.070 \\
\hline DZ5 & 4 & 0.636 & 0.333 & 0.500 & 4 & 0.682 & 0.500 & 0.286 & 4 & 0.712 & 0.500 & 0.318 \\
\hline DZ6 & 3 & 0.530 & 0.667 & -0.290 & 2 & 0.485 & 0.333 & 0.333 & 3 & 0.621 & 0.333 & 0.487 \\
\hline DZ7 & 3 & 0.682 & 0.833 & -0.250 & 2 & 0.303 & $0.000^{*}$ & 1.000 & 4 & 0.561 & 0.667 & -0.212 \\
\hline DZ8 & 4 & 0.742 & 0.833 & -0.136 & 3 & 0.682 & $0.167^{*}$ & 0.773 & 2 & 0.546 & $0.000^{*}$ & 1.000 \\
\hline DZ9 & 4 & 0.636 & 0.833 & -0.351 & 2 & 0.303 & 0.333 & -0.111 & 3 & 0.621 & $0.167^{*}$ & 0.750 \\
\hline DZ10 & 5 & 0.788 & 0.333 & 0.600 & 3 & 0.621 & 0.667 & -0.081 & 3 & 0.667 & $0.000^{*}$ & 1.000 \\
\hline DZ11 & 5 & 0.758 & 0.500 & 0.362 & 4 & 0.727 & 0.667 & 0.091 & 4 & 0.636 & 0.500 & 0.231 \\
\hline$D Z 12$ & 2 & 0.485 & 0.667 & -0.429 & 5 & 0.727 & 0.833 & -0.163 & 2 & 0.167 & 0.167 & 0.000 \\
\hline$D Z 13$ & 5 & 0.803 & 0.833 & -0.042 & 3 & 0.667 & 0.667 & 0.000 & 5 & 0.789 & 0.833 & -0.064 \\
\hline DZ14 & 2 & 0.530 & 0.500 & 0.063 & 3 & 0.667 & 1.000 & -0.579 & 2 & 0.546 & $1.000^{*}$ & -1.000 \\
\hline DZ15 & 3 & 0.439 & 0.333 & 0.259 & 3 & 0.439 & 0.500 & -0.154 & 4 & 0.561 & 0.500 & 0.118 \\
\hline DZ16 & 2 & 0.167 & 0.167 & 0.000 & 2 & 0.167 & 0.167 & 0.000 & 1 & 0.000 & 0.000 & - \\
\hline
\end{tabular}

$\mathrm{N}=$ number of individuals tested; $N_{\mathrm{A}}=$ number of alleles; $H_{\mathrm{E}}=$ expected heterozygosity; $H_{\mathrm{O}}=$ observed heterozygosity; $F_{\mathrm{IS}}=$ inbreeding coefficient; *Significant deviation from Hardy-Weinberg equilibrium; - = not surveyed. 


\section{ACKNOWLEDGMENTS}

Research supported by the Postdoctoral Science Foundation of China (\#13A180057) and the National Natural Science Foundation of China (\#31100272).

\section{REFERENCES}

Chen X, Sang X, Li S, Zhang S, et al. (2010). Studies on a chlorogenic acid-producing endophytic fungi isolated from Eucommia ulmoides Oliver. J. Ind. Microbiol. Biotechnol. 37: 447-454.http://dx.doi.org/10.1007/s10295-010-0690-0

Deng J, Liu Y and Huang H (2006). Development and characterization of microsatellite markers in Eucommia ulmoides Oliver (Eucommiaceae). Mol. Ecol. Notes 6: 496-498. http://dx.doi.org/10.1111/j.1471-8286.2006.01287.x

Fu ZZ, Li YH and Li Y (2014). Isolation and characterization of microsatellite markers for Forsythia suspensa (Thunb.) Vahl (Oleaceae) using 454 sequencing technology. Biochem. Syst. Ecol. 57: 328-331. http://dx.doi.org/10.1016/j. bse.2014.09.012

Goudet J (1995). FSTAT (version 1.2): A computer software to calculate F-statistics. J. Hered. 86: 485-486.

Hall LA and Beissinger SR (2014). A practical toolbox for design and analysis of landscape genetics studies. Landscape Ecol. 29: 1487-1504. http://dx.doi.org/10.1007/s10980-014-0082-3

Hussain M, Hussain MT, Rama NH, Hameed S, et al. (2003). Synthesis and antimicrobial activities of some isocoumarin and dihydroisocoumarin derivatives. Nat. Prod. Res. 17: 207-214.http://dx.doi.org/10.1080/1057563021000040826

Li Y, Zhang W, Fu ZZ, Wang W, et al. (2015). Isolation and characterization of microsatellite markers for Achyranthesbidentata (Amaranthaceae) using next-generation sequencing platform. Biochem. Syst. Ecol. 61: 437440. http://dx.doi.org/10.1016/j.bse.2015.06.032

Li YC, Korol AB, Fahima T, Beiles A, et al. (2002). Microsatellites: genomic distribution, putative functions and mutational mechanisms: a review. Mol. Ecol. 11: 2453-2465.http://dx.doi.org/10.1046/j.1365-294X.2002.01643.X

Rousset F (2008). genepop'007: a complete re-implementation of the genepop software for Windows and Linux. Mol. Ecol. Resour. 8: 103-106.http://dx.doi.org/10.1111/j.1471-8286.2007.01931.x

Rozen S and Skaletsky HJ (2000). Primer3 on the WWW for general users and for biologist programmers. In: Bioinformatics methods and protocols: methods in molecular biology (Krawetz S and Misener S, eds.) Humana Press, Totowa, USA, 365-386.

Thiel T, Michalek W, Varshney RK and Graner A (2003). Exploiting EST databases for the development and characterization of gene-derived SSR-markers in barley (Hordeum vulgare L.). Theor. Appl. Genet. 106: 411-422.

Wang AQ, Huang LQ, Shao AJ, Cui GH, et al. (2006). [Genetic diversity of Eucommia ulmoides by RAPD analysis]. Zhongguo Zhong Yao Za Zhi 31: 1583-1586.

Wei N, Dick CW, Lowe AJ and Gardner MG (2013). Polymorphic microsatellite loci for Virola sebifera (Myristicaceae) derived from shotgun 454 pyrosequencing. Appl. Plant Sci. 1: 1200295.http://dx.doi.org/10.3732/apps.1200295

Yao XH, Deng JY and Huang HW (2012). Genetic diversity in Eucommia ulmoides (Eucommiaceae), an endangered traditional Chinese medicinal plant. Conserv. Genet. 13: 1499-1507. http://dx.doi.org/10.1007/s10592-012-0393-3

Yeh FC, Yang R, Boyle TJ, Ye Z, et al. (2000). PopGene 32. Microsoft Window-based freeware for population genetic analysis, version 1.32. Molecular Biology and Biotechnology Centre, University of Alberta, Edmonton, Canada. 